\title{
SILVER COMPLEXES FOR TUBERCULOSIS TREATMENT: A SHORT REVIEW
}

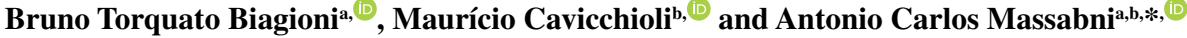 \\ aPrograma de Pós-Graduação em Biotecnologia em Medicina Regenerativa e Química Medicinal, Universidade de Araraquara, \\ 14801-340 Araraquara - SP, Brasil Universidade de Araraquara, 14801-340 Araraquara - SP, Brasil \\ 'Instituto de Química, Universidade Estadual Paulista “Júlio de Mesquita Filho", 14800-060 Araraquara - SP, Brasil
}

Recebido em 02/03/2021; aceito em 21/07/2021; publicado na web em 20/08/2021

\begin{abstract}
This paper is a brief review of silver complexes that have been identified as antibacterial drugs with promising use for the treatment of tuberculosis (TB). This treatment aims to cure and discontinue the transmission of the disease. The excessive and inappropriate use of drugs has jeopardized the effectiveness of antibiotics for the TB treatment, bringing bacterial resistance to them. To achieve these objectives, the drugs used must be able to eliminate rapidly the bacterial population, avoiding the selection of drug-resistant strains and preventing its recurrence. The number of new cases of multidrug-resistant TB (MDR-TB) and extensively drug-resistant TB (XDR-TB) continues to rise. So, it is important to develop more research to introduce new drugs or improve existing ones, which can reduce the treatment time, increasing drug adherence and reducing MDR-TB and XDR-TB. The Ag(I) complexes were described with different types of ligands, and bonds to $\mathrm{Ag}(\mathrm{I})$ occur by $\mathrm{N}, \mathrm{O}, \mathrm{P}$, and $\mathrm{S}$ atoms. Metal complexes are presented as options for antiTB treatments. There are no cases of TB treatments using metal complexes but research in this area show that they could be used in the future to eradicate the bacteria that contaminate environments, surgical materials and other objects.
\end{abstract}

Keywords: Mycobacterium tuberculosis; silver(I) compounds; drugs.

\section{INTRODUCTION}

Tuberculosis (TB) is one of the ten leading causes of human deaths, the most lethal caused by a single infectious agent (above HIV/AIDS). This disease affects $1 / 3$ of the world's population and approximately 10 million people develop TB each year, resulting in 2 million deaths.

Although standard medical treatment with TB drugs is highly effective, more therapies are necessary to reduce the number of infectious cases. The number of new cases of multidrug-resistant TB (MDR-TB) and extensively drug-resistant TB (XDR-TB) continues to increase. ${ }^{2}$ Understanding TB epidemiological profile is fundamental to reduce the time between the first symptoms, the diagnosis and the beginning of supervised medical treatments. It is also important to develop researches to introduce new drugs or improve the already existent, which can reduce the treatment time from 6 to 3 months, for example, therefore avoiding abandonment and increasing adherence, which prevent MDR-TB and XDR-TB.

While some initiatives against TB aim to eradicate social conditions that increase TB cases (such as extreme poverty, malnutrition and smoking), other actions seek to increase the population's immunity to the bacteria via Bacillus Calmette-Guérin (BCG) vaccine; there are also numerous efforts to eliminate Mycobacterium tuberculosis as cause of the active (and lethal) form of TB, creating new drugs for potential use. This paper also presents the state of the art in the research of metal complexes as possible drugs for treatment of TB. ${ }^{1-3}$

\section{Drugs used in the treatment of TB}

Standard treatment for TB consists of four first-line drugs: isoniazid, rifampicin, pyrazinamide and ethambutol, and resistance of bacilli can occur to all first-line drugs. ${ }^{3}$ Tuberculosis treatment aims to cure (preventing its morbidity and mortality) and to discontinue the disease transmission (making patients noninfectious). To achieve these objectives, the drugs used should be capable of rapidly eliminating the bacterial population, preventing the selection of drug-resistant strains (consequently, the appearance of MDR-TB and XDR-TB) and sterilizing the lesion, preventing its recurrence. ${ }^{4}$

Since 1960, in the face of bacterial resistance and the increase in cases of TB deaths, the treatment regimen has been standardized and the current recommended treatment for cases of drug-susceptible TB is a six-month regimen with the four first-line drugs. ${ }^{4}$ The structural formulas of these substances are shown in Figure 1.<smiles>NNC(=O)c1ccncc1</smiles>

isoniazid<smiles>NC(=O)c1cnccn1</smiles>

pyrazinamide

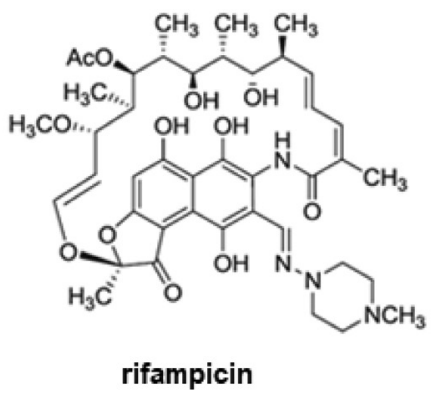<smiles>CCC(CO)NCCNC(CC)CO</smiles>

ethambutol

Figure 1. Structural formulas of the most used substances for TB treatment

The second-line drugs (Table 1) are used for the treatment of rifampicin-resistant $\mathrm{TB}$ and MDR-TB and can be classified as fluoroquinolones (Group A), aminoglycosides (Group B), other main agents (Group C) and additional agents (Group D). ${ }^{5}$ 
Table 1. Classes of second line anti-TB drugs

\begin{tabular}{lcc}
\hline Group & Classification & Drugs \\
\hline Group A & fluoroquinolones & $\begin{array}{c}\text { levofloxacin, moxifloxacin, } \\
\text { gatifloxacin }\end{array}$ \\
\hline Group B & aminoglycosides & $\begin{array}{c}\text { amikacin, capreomycin, } \\
\text { kanamycin, streptomycin }\end{array}$ \\
\hline Group C & $\begin{array}{c}\text { other important } \\
\text { second-line agents }\end{array}$ & $\begin{array}{c}\text { ethionamide / protionamide, } \\
\text { cycloserine / terizidone, linezolid, } \\
\text { clofazimine }\end{array}$ \\
\hline Group D & additional agents & $\begin{array}{c}\text { D1: pyrazinamide, ethambutol, } \\
\text { high dose of isoniazid } \\
\text { D2: bedaquiline, delamanid } \\
\end{array}$ \\
& & $\begin{array}{c}\text { D3: para-aminosalicylic acid, } \\
\text { imipenem-cilastatin, meropenem, } \\
\text { amoxicillin-clavulanate, thioketone }\end{array}$ \\
\hline
\end{tabular}

\section{Drug resistance}

The rapid emergence of resistant bacteria has jeopardized the effectiveness of antibiotics for TB treatment. The crisis of antibiotic resistance has been attributed to the excessive and inappropriate use of these drugs, as well as to the lack of development of new drugs by the pharmaceutical industry, due to the reduction of economic incentives and challenging regulatory requirements. ${ }^{6}$

Bacterial genes can be inherited or can be acquired from plasmids. Such horizontal gene transfer may allow the transfer of antibiotic resistance among different types of bacteria. Resistance can also appear through mutation. Antibiotics can remove drug-sensitive competitors, permitting resistant bacteria to reproduce as a result of a natural selection. ${ }^{6}$

Mycobacteria have some strategies for developing drug resistance. The main ones are: decreased permeability of the cell wall, increased efflux of the drug, degradation or inactivation of the drugs through enzymes and genetic modifications in the target of the drug. In M. tuberculosis, resistance is acquired only by chromosomal mutations and multiresistance occurs due to the accumulation of mutations in independent genes, which are each responsible for resistance to a particular antibiotic. ${ }^{7}$

Mycobacterium tuberculosis resistance types are classified as natural, initial, primary and acquired (or secondary). Natural resistance occurs with spontaneous mutation of bacilli; the initial one is observed in patients with some form of resistance to one or more drugs whose history of treatment is unknown; primary resistance occurs in patients infected with a resistant $M$. tuberculosis strain and the acquired or secondary occurs due to inadequate use of prescription drugs. ${ }^{7}$

\section{Multidrug resistance}

Treatment of multidrug-resistant TB is one of the greatest global challenges for TB control, especially rifampicin-resistant, isolated or combined with other drugs, as it is the most effective drug against $M$. tuberculosis bacillus. Schemes without rifampicin in its composition, either by resistance or intolerance, require the use of second-line drugs, which results in treatment with a longer duration, with great potential for toxicity and bad prognosis. ${ }^{8}$

As for drug-sensitive TB cases, the therapeutic regimen for MDRTB should use at least four effective drugs (never used before or with a high likelihood of being sensitive), according to the rational drug classification, containing at least two essential drugs (bactericidal and sterilizing), plus two accompanying drugs (protective action against essential acquired resistance). ${ }^{8}$
In patients with rifampicin-resistant tuberculosis or MDR-TB, therapy with at least five anti-TB drugs during the intensive phase, including pyrazinamide and four second-line drugs (one from Group A, one from Group B and at least two from Group C) is recommended. If the minimum number of drugs against TB cannot be included in the treatment dose as indicated above, one agent of Group D2 and other agents of Group D3 can be added to reach the total of five (Table 2). ${ }^{5}$

\section{SILVER USES}

Silver and its silver(I) salts are considered toxic for humans as well to lower organisms. Many formulations included silver(I) compounds as drugs for certain medical treatments as antimicrobial and anticancer agents were used in the past century. ${ }^{9}$

The metal does not have biological role. On the contrary, it is considered highly toxic for living organisms depending on its concentration. Nevertheless, it is not a cumulative poison in the body, in a different way of other heavy metals. For some time now, $\mathrm{Ag}(\mathrm{I})$ complexes have been regarded as possible metallopharmaceuticals due to their biological properties. Several studies show the activities of silver against bacteria, fungi, parasites, cancers, and malaria. ${ }^{9}$

According to Medici et al., ${ }^{9}$ the efficacy of $\mathrm{Ag}(\mathrm{I})$ complexes against bacteria, primarily (the focus of this short review), depends on a number of factors: lipophilicity, redox propensity, water solubility and stability, and release rate of the $\operatorname{Ag}(\mathrm{I})$ ions. However, the authors comment that even if an $\mathrm{Ag}(\mathrm{I})$ complex is well-designed for medical use, its efficacy may decrease considerably when used in vivo because of formation of insoluble $\mathrm{AgCl}$ or binding of the complex to cellular enzymes.

A common approach for the syntheses of metal complexesincluding $\operatorname{Ag}(\mathrm{I})$ complexes - is to associate the action of the metal center with that of a drug that is already in use or has already exhibited therapeutic properties against a certain disease. The intention is that the combination of metal and ligand will enhance the performance of the resulting complex, as is the case a number of $\operatorname{Ag}(\mathrm{I})$ silver complexes discussed in this article.

\section{METHODOLOGY}

This work is an integrative review of the literature, elaborated from the delimitation of the theme and the guiding question, formulation of objectives, definition of inclusion and exclusion criteria, identification, selection, categorization and evaluation of studies, interpretation of results and presentation of the known syntheses. ${ }^{10}$

Data collection occurred in December 2020 and was redone between $4^{\text {th }}$ and $8^{\text {th }}$ May 2021. Searches were performed on SciELO, PubMed, Google Scholar and Capes Portal de Periódicos, using the descriptors: "silver", "tuberculosis", "ethambutol”, "pyrazinamide”, "rifampicin", "isoniazid" and "silver complex".

As inclusion criteria for the selection of the studies of this integrative review, articles published between 2010 and 2020, in national and international journals, written in English, addressing the synthesis, characterization and in vitro biological analysis of $\mathrm{Ag}(\mathrm{I})$ complexes were reviewed. The following texts were excluded from the study: editorials, theses, dissertations, books, congress abstracts, review studies, articles that did not fit into the time frame or did not meet the proposed objective. Articles found in more than one database were considered only once.

The time frame from 2010 to 2020 is justified by the need to seek updated evidence on the subject in question. Systematic reviews found during the search procedure were not included in the presentation of results but were used to deepen the discussion of this study. Table 2 
Table 2. Bibliographic search strategy

\begin{tabular}{|c|c|c|}
\hline Search string & Databases & Search results \\
\hline "silver" AND "tuberculosis" AND "ethambutol" & PubMed & 38 articles \\
\hline "silver" AND "tuberculosis" AND "pyrazinamide" & SciELO & 8 articles \\
\hline "silver" AND "tuberculosis" AND "isoniazid" & Google Scholar & 14,148 articles \\
\hline "silver complex" AND “tuberculosis" & Capes Portal de Periódicos & 1,661 articles \\
\hline TOTAL & & 15,855 articles \\
\hline
\end{tabular}
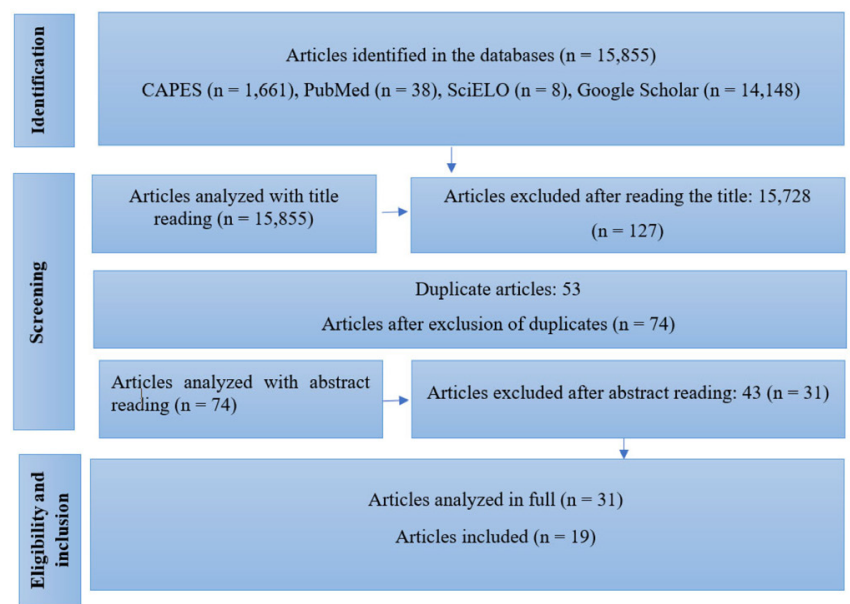

Figure 2. Flowchart illustrating the study selection process according to the PRISMA recommendation

shows bibliographic search strategy and Figure 2 shows flowchart illustrating the study selection process according to the PRISMA recommendation. ${ }^{11}$
The analysis process began with evaluation of the title, followed by the reading of the summaries of the publications in order to verify if they contemplated the research question and if they met the established inclusion criteria. After the preselection, the studies were read in their entirety in order to avoid selection bias. The final sample consisted of 19 selected articles.

To collect information relevant to the study, a literature review protocol was used, and an Excel software database was prepared with the information: author(s), year of publication, journal of publication, database, ligands and metal complexes.

The synthesis and critical analysis of the results are presented in the next section.

\section{RESULTS AND DISCUSSION}

The results of the search were analyzed and arranged in Table 3.

In a general way all 55 complexes described in the 19 articles are relatively easy to prepare, since experimental conditions are not special.

There are complexes where $\operatorname{Ag}(\mathrm{I})$ is coordinated to only one type

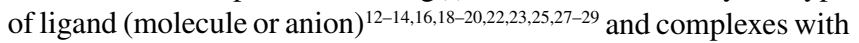
mixed ligands. ${ }^{15,17,21,24,26,30}$

$\mathrm{Ag}(\mathrm{I})$ is coordinated preferentially to $\mathrm{N}-{ }^{12,14-20,22,23,26-28,30}$ and

Table 3. Results of the search

\begin{tabular}{|c|c|c|c|}
\hline $\begin{array}{l}\text { Title of the articles, authors, year of publication and } \\
\text { reference }\end{array}$ & Ligands & Silver(I) complex & $\begin{array}{l}\text { Anti-TB activity, } \mathrm{MIC}_{90} \\
\left.\mu \mathrm{g} \mathrm{mL} \mathrm{mL}^{-1}(\mu \mathrm{mol} \mathrm{L})^{-1}\right)\end{array}$ \\
\hline $\begin{array}{l}\mathrm{Pt}(\mathrm{II}) \text { and } \operatorname{Ag}(\mathrm{I}) \text { complexes with acesulfame: crystal } \\
\text { structure and a study of their antitumoral, antimicrobial } \\
\text { and antiviral activities, Cavicchioli } \text { et al. }(2010)^{12}\end{array}$ & acesulfame (ace), $\mathrm{C}_{4} \mathrm{H}_{4} \mathrm{KNO}_{4} \mathrm{~S}$ & {$\left[\mathrm{Ag}\left(\mathrm{C}_{4} \mathrm{H}_{4} \mathrm{NO}_{4} \mathrm{~S}\right)\right]_{n}$} & $\mathrm{n} / \mathrm{s}(3.1)^{\dagger}$ \\
\hline $\begin{array}{l}\text { 6-Mercaptopurine complexes with silver and gold ions: } \\
\text { Anti-tuberculosis and anti-cancer activities, Cuin et al. } \\
(2011)^{13}\end{array}$ & 6-mercaptopurine, $\mathrm{C}_{5} \mathrm{H}_{4} \mathrm{~N}_{4} \mathrm{~S}$ & $\left(\mathrm{Ag}\left[\mathrm{C}_{5} \mathrm{H}_{3} \mathrm{~N}_{4} \mathrm{~S}\right]\right) \cdot \mathrm{H}_{2} \mathrm{O}$ & $25.0(93.2)$ \\
\hline $\begin{array}{l}\text { A broad study of two new promising antimycobacterial } \\
\text { drugs: } \operatorname{Ag}(\mathrm{I}) \text { and } \mathrm{Au}(\mathrm{I}) \text { complexes with } 2 \text {-(2-thienyl) } \\
\text { benzothiazole, Pereira } \text { et al. }(2012)^{14}\end{array}$ & 2-(2-thienyl)benzothiazole, $\mathrm{C}_{11} \mathrm{H}_{7} \mathrm{NS}_{2}$ & {$\left[\mathrm{Ag}\left(\mathrm{C}_{11} \mathrm{H}_{7} \mathrm{NS}_{2}\right)_{2} \mathrm{NO}_{3}\right]$} & $12.5(20.7)$ \\
\hline $\begin{array}{l}\text { Synthesis, crystal structures, antimicrobial, antifungal } \\
\text { and antituberculosis activities of mixed ligand silver(I) } \\
\text { complexes, Altaf et al. }(2013)^{15}\end{array}$ & $\begin{array}{l}\text { (P1): triphenyl phosphine } \\
\text { (P2): tricyclohexyl phosphine } \\
\text { (P3): phenyldicyclohexyl phosphine } \\
\text { (P4): diphenylcyclohexyl phosphine } \\
\text { (P5): diphenyl(p-tolyl) phosphine } \\
\\
\text { (L1): thiosemicarbazide } \\
\text { (L2): 2-(propan-2-ylidene) } \\
\text { hydrazinecarbothioamide } \\
\text { (L3): 4,5-dihydrothiazole-2-thiol (A) or } \\
\text { thiazolidine-2-thione (B) }\end{array}$ & $\begin{array}{c}1=\left[\mathrm{Ag}_{2}(\mathrm{P} 1)_{2}(\mathrm{~L} 1)_{4}\right]\left(\mathrm{NO}_{3}\right) \cdot 2 \mathrm{H}_{2} \mathrm{O} \\
2=\left[\mathrm{Ag}_{2}(\mathrm{P} 1)_{2}(\mathrm{~L} 3)_{4}\right]\left(\mathrm{NO}_{3}\right)_{2} \\
3=\left[\mathrm{Ag}(\mathrm{P} 2)(\mathrm{L} 3)_{2}\right]\left(\mathrm{NO}_{3}\right) \\
4=\left[\mathrm{Ag}(\mathrm{P} 2)(\mathrm{L} 1)_{2}\right] \mathrm{PF}_{6} \\
\left.5=\left[\mathrm{Ag}(\mathrm{P} 3)_{2}(\mathrm{~L} 2)\right]_{2}\left(\mathrm{NO}_{3}\right)\right\} \mathrm{NO}_{3} \\
6=\left[\mathrm{Ag}_{2}(\mathrm{P} 2)_{2}(\mathrm{~L} 3)_{2}\right](\mathrm{PF} 6)_{2} \\
7=\left[\mathrm{Ag}_{2}(\mathrm{P} 4)_{2}(\mathrm{~L} 2)_{2}\right]\left(\mathrm{ClO}_{4}\right)_{2} \\
8=\left[\mathrm{Ag}(\mathrm{P} 5)_{2}(\mathrm{~L} 2)(\mathrm{Br})\right] \\
9=\left[\mathrm{Ag}_{2}(\mathrm{P} 1)_{2}(\mathrm{~L} 2)_{2}\right]\left(\mathrm{NO}_{3}\right)_{2}\end{array}$ & $\begin{array}{c}1=10.2(8.1) \\
2=15.6(11.6) \\
3=10.6(14.7) \\
4=10.6(14.2) \\
5=12.5(7.35) \\
6=7.8(6.0) \\
7=12.5(10.3) \\
8=12.5(14.3) \\
9=10.2(9.0)\end{array}$ \\
\hline $\begin{array}{l}\text { Silver(I) complexes with symmetrical Schiff bases: } \\
\text { Synthesis, structural characterization, DFT studies and } \\
\text { antimycobacterial assays, Paiva et al. }(2013)^{16}\end{array}$ & $\begin{array}{c}\text { MBDA }=N, N^{\prime} \text {-bis [(4-methoxyphenyl) } \\
\text { methylidene }] \text { ethane-1,2-diamine, } \\
\qquad \mathrm{C}_{18} \mathrm{H}_{20} \mathrm{~N}_{2} \mathrm{O}_{2} \\
\text { MBDB }=N, N^{\prime} \text {-bis[(4-methoxyphenyl) } \\
\text { methylidene]propane-1,3-diamine, } \\
\mathrm{C}_{19} \mathrm{H}_{22} \mathrm{~N}_{2} \mathrm{O}_{2} \\
\end{array}$ & $\begin{array}{c}\operatorname{AgMBDA}=\left[\mathrm{Ag}\left(\mathrm{C}_{18} \mathrm{H}_{20} \mathrm{~N}_{2} \mathrm{O}_{2}\right)_{2}\right] \mathrm{NO}_{3} \\
\operatorname{AgMBDB}=\left[\mathrm{Ag}\left(\mathrm{C}_{19} \mathrm{H}_{22} \mathrm{~N}_{2} \mathrm{O}_{2}\right) \mathrm{NO}_{3}\right] \cdot 1 / 2 \mathrm{H}_{2} \mathrm{O}\end{array}$ & $\begin{array}{l}\operatorname{AgMBDA}=21.2(27.8) \\
\operatorname{AgMBDB}=11.3(23.5)\end{array}$ \\
\hline $\begin{array}{l}\text { Synthesis and biological evaluation of ternary silver com- } \\
\text { pounds bearing } \mathrm{N}, \mathrm{N} \text {-chelating ligands and thiourea: } \mathrm{X}-\text {-ray } \\
\text { structure of }\left[\{\mathrm{Ag}(\mathrm{bpy})(\mu-\mathrm{tu})\}_{2}\right]\left(\mathrm{NO}_{3}\right)_{2}\left(\mathrm{bpy}=2,2^{\prime} \text {-bipyri- }\right. \\
\text { dine; tu = thiourea), Segura } \text { et al. }(2014)^{17}\end{array}$ & $\begin{array}{c}\text { phen }=1,10 \text {-phenanthroline } \\
\text { bpy }=2,20 \text {-bipyridine } \\
\text { tu }=\text { thiourea }\end{array}$ & $\begin{array}{c}1=\left[\{\mathrm{Ag}(\text { phen })(\mu-\mathrm{tu})\}_{2}\right]\left(\mathrm{NO}_{3}\right)_{2} \\
\mathrm{C}_{26} \mathrm{H}_{24} \mathrm{Ag}_{2} \mathrm{~N}_{10} \mathrm{O}_{6} \mathrm{~S}_{2} \\
2=\left[\{\mathrm{Ag}(\text { phen })(\mu-\mathrm{tu})\}_{2}\right]\left(\mathrm{CF}_{3} \mathrm{SO}_{3}\right)_{2} \\
\mathrm{C}_{28} \mathrm{H}_{24} \mathrm{Ag}_{2} \mathrm{~F}_{6} \mathrm{~N}_{8} \mathrm{O}_{6} \mathrm{~S}_{4} \\
3=\left[\{\mathrm{Ag}(\text { bpyy })(\mu-\mathrm{tu})\}_{2}\right]\left(\mathrm{NO}_{3}\right)_{2} \\
\mathrm{C}_{22} \mathrm{H}_{24} \mathrm{Ag}_{2} \mathrm{~N}_{10} \mathrm{O}_{6 \mathrm{~S}} 2\end{array}$ & $\begin{array}{c}1=4.7 \pm 0.4(11.0 \pm 1.0) \\
2=7.3 \pm 1.4(14.2 \pm 2.8) \\
3>25\end{array}$ \\
\hline
\end{tabular}


Table 3. Results of the search (cont.)

Title of the articles, authors, year of publication and reference

Coordinative versatility of a Schiff base containing thiophene: Synthesis, characterization and biological activity of zinc (II) and silver (I) complexes, Silva et al. $(2014)^{18}$

A silver complex with cycloserine: synthesis, spectroscopic characterization, crystal structure and in vitro biological studies, Ciol et al. (2018) ${ }^{19}$

Silver(I) and zinc(II) complexes with symmetrical cinnamaldehyde Schiff base derivative: Spectroscopic, powder diffraction characterization, and antimycobacterial studies, Amaral et al. $(2018)^{20}$

Enhancement in anti-tubercular activity of indole based thiosemicarbazones on complexation with copper(I) and silver(I) halides: Structure elucidation, evaluation and molecular modelling, Khan et al. $(2018)^{21}$

Synthesis and antimicrobial activity of a phenanthrolineisoniazid hybrid ligand and its $\mathrm{Ag}^{+}$and $\mathrm{Mn}^{2+}$ complexes, Ahmed et al. $(2019)^{22}$

\section{Ligands}

ThioEn $=N, N^{\prime}$-bis $($ thiophen-2-

ylmethylene)ethane-1,2-diamine

cycloserine, $\mathrm{C}_{3} \mathrm{H}_{6} \mathrm{~N}_{2} \mathrm{O}_{2}$
Silver(I) complex

$1=[\mathrm{Ag}(\mathrm{ThioEn})] \mathrm{NO}_{3}$

$2=\left[\mathrm{Ag}(\text { ThioEn })_{2}\right] \mathrm{NO}_{3}$
Anti-TB activity, $\mathrm{MIC}_{90}$ $\mu \mathrm{g} \mathrm{mL}-1(\mu \mathrm{mol} \mathrm{L}-1)$

$$
1=12.2(29.2)
$$$$
2=24.5(36.7)
$$

$N, N$ '-bis(trans-cinnamaldehyde)ethane-

-1,2-diamine $\quad\left[\mathrm{C}_{20} \mathrm{H}_{20} \mathrm{AgN}_{3} \mathrm{O}_{3}\right]$

$16.5 \pm 0.6(79.1)$

Silver complexes with fluoroanthranilic acid isomers: spectroscopic characterization, antimycobacterial activity and cytotoxic studies over a panel of tumor cells, Manzano et al. (2019) ${ }^{23}$

$\mathrm{C}_{20} \mathrm{H}_{20} \mathrm{~N}_{2}$

$\left[\mathrm{AgC}_{3} \mathrm{H}_{5} \mathrm{~N}_{2} \mathrm{O}_{2}\right]$
fluoroanthranilic acid
$\mathrm{C}_{7} \mathrm{H}_{6} \mathrm{FNO}_{2}$

thiosemicarbazones $\left(\mathrm{H}^{1} \mathrm{~L}, \mathrm{H}^{2} \mathrm{~L}, \mathrm{H}^{3} \mathrm{~L}\right)+$ phosphine

$\begin{array}{cc}13=\left[\mathrm{AgCl}(\eta 1-\mathrm{S}-\mathrm{HIntsc})\left(\mathrm{Ph}_{3} \mathrm{P}\right)_{2}\right] & 13=1.6(\mathrm{n} / \mathrm{s})^{\dagger \dagger} \\ 14=\left[\mathrm{AgBr}(\eta 1-\mathrm{S}-\mathrm{HIntsc})\left(\mathrm{Ph}_{3} \mathrm{P}\right)_{2}\right] & 14=6.3(\mathrm{n} / \mathrm{s}) \\ 15=\left[\mathrm{AgCl}(\eta 1-\mathrm{S}-\mathrm{HIntsc}-\mathrm{N} 1-\mathrm{Me})\left(\mathrm{Ph}_{3} \mathrm{P}\right)_{2}\right] & 15=1.6(\mathrm{n} / \mathrm{s}) \\ 16=\left[\mathrm{AgBr}(\eta 1-\mathrm{S}-\mathrm{HIntsc}-\mathrm{N} 1-\mathrm{Me})\left(\mathrm{Ph}_{3} \mathrm{P}\right)_{2}\right] & 16=1.6(\mathrm{n} / \mathrm{s}) \\ 17=\left[\mathrm{AgCl}(\eta 1-\mathrm{S}-5-\mathrm{MeOHIntsc})\left(\mathrm{Ph}_{3} \mathrm{P}\right)_{2}\right] & 17=1.6(\mathrm{n} / \mathrm{s}) \\ 18=\left[\mathrm{AgBr}\left(\eta 1-\mathrm{S}-5-\mathrm{MeOHIntsc}^{\prime}\left(\mathrm{Ph}_{3} \mathrm{P}\right)_{2}\right]\right. & 18=1.6(\mathrm{n} / \mathrm{s}) \\ 1=\left[\mathrm{Ag}(\mathbf{L})_{2}\right] \mathrm{NO}_{3} \cdot 2 \mathrm{H}_{2} \mathrm{O} & 1=2.5(2.9) \\ {\left[\mathrm{Ag}\left(\mathrm{C}_{18} \mathrm{H}_{11} \mathrm{~N}_{5} \mathrm{O}_{2}\right)_{2}\right] \mathrm{NO}_{3} \cdot 2 \mathrm{H}_{2} \mathrm{O}} & \\ 2=\left[\mathrm{Ag}(\mathbf{L})_{2}\right] \mathrm{BF}_{4} & 2=2.5(2.9) \\ {\left[\mathrm{Ag}\left(\mathrm{C}_{18} \mathrm{H}_{11} \mathrm{~N}_{5} \mathrm{O}_{2}\right)_{2}\right] \mathrm{BF}_{4}} & \end{array}$

\begin{tabular}{cc}
$13=\left[\mathrm{AgCl}(\eta 1-\mathrm{S}-\mathrm{HIntsc})\left(\mathrm{Ph}_{3} \mathrm{P}\right)_{2}\right]$ & $13=1.6(\mathrm{n} / \mathrm{s})^{\dagger \dagger}$ \\
$14=\left[\mathrm{AgBr}(\eta 1-\mathrm{S}-\mathrm{HIntsc})\left(\mathrm{Ph}_{3} \mathrm{P}\right)_{2}\right]$ & $14=6.3(\mathrm{n} / \mathrm{s})$ \\
$15=\left[\mathrm{AgCl}(\eta 1-\mathrm{S}-\mathrm{HIntsc}-\mathrm{N} 1-\mathrm{Me})\left(\mathrm{Ph}_{3} \mathrm{P}\right)_{2}\right]$ & $15=1.6(\mathrm{n} / \mathrm{s})$ \\
$16=\left[\mathrm{AgBr}(\eta 1-\mathrm{S}-\mathrm{HIntsc}-\mathrm{N} 1-\mathrm{Me})\left(\mathrm{Ph}_{3} \mathrm{P}\right)_{2}\right]$ & $16=1.6(\mathrm{n} / \mathrm{s})$ \\
$17=\left[\mathrm{AgCl}(\eta 1-\mathrm{S}-5-\mathrm{MeOHIntsc})\left(\mathrm{Ph}_{3} \mathrm{P}\right)_{2}\right]$ & $17=1.6(\mathrm{n} / \mathrm{s})$ \\
$18=\left[\mathrm{AgBr}(\eta 1-\mathrm{S}-5-\mathrm{MeOHIntsc})\left(\mathrm{Ph}_{3} \mathrm{P}\right)_{2}\right]$ & $18=1.6(\mathrm{n} / \mathrm{s})$ \\
\hline $1=\left[\mathrm{Ag}(\mathbf{L})_{2}\right] \mathrm{NO}_{3} \cdot 2 \mathrm{H}_{2} \mathrm{O}$ & $1=2.5(2.9)$ \\
{$\left[\mathrm{Ag}\left(\mathrm{C}_{18} \mathrm{H}_{11} \mathrm{~N}_{5} \mathrm{O}_{2}\right)_{2}\right] \mathrm{NO}_{3} \cdot 2 \mathrm{H}_{2} \mathrm{O}$} & \\
$2=\left[\mathrm{Ag}(\mathbf{L})_{2}\right] \mathrm{BF}_{4}$ & $2=2.5(2.9)$ \\
{$\left[\mathrm{Ag}\left(\mathrm{C}_{18} \mathrm{H}_{11} \mathrm{~N}_{5} \mathrm{O}_{2}\right)_{2}\right] \mathrm{BF}_{4}$} &
\end{tabular}

$\left[\mathrm{Ag}\left(\mathrm{C}_{7} \mathrm{H}_{6} \mathrm{FNO}_{2}\right)_{2}\right]^{+}$isomers (4fa, $5 \mathrm{fa}$ and $6 \mathrm{fa}$ )
$\mathrm{Ag} 4 \mathrm{fa}=2.6 \pm 0.1(9.8 \pm 0.5)$

$\mathrm{Ag} 5 \mathrm{fa}=4.2 \pm 2.2(15.9 \pm 8.6)$

Ag6fa $=2.6 \pm 3.1(9.8 \pm 11.9)$
Cytotoxic and apoptotic effects of ternary silver (I) complexes bearing 2-formylpyridine thiosemicarbazones and 1,10-phenanthroline, Silva et al. $(2020)^{24}$
2-formylpyridine-N(4)-Rthiosemicarbazones (L) and 1,10-phenanthroline (phen)

$$
\begin{gathered}
1=[\mathrm{Ag}(\text { phen })(\mathrm{PHTSC})]\left(\mathrm{NO}_{3}\right) \cdot \mathrm{HCl} \cdot 2 \mathrm{H}_{2} \mathrm{O} \\
\mathrm{C}_{19} \mathrm{H}_{21} \mathrm{AgClN}_{7} \mathrm{O}_{5} \mathrm{~S} \\
2=\left[\mathrm{Ag}(\text { phen })\left(\mathrm{PMTSC}^{2}\right)\right]\left(\mathrm{NO}_{3}\right) \cdot \mathrm{HCl} \cdot \mathrm{CH}_{3} \mathrm{OH} \\
\mathrm{C}_{21} \mathrm{H}_{23} \mathrm{AgClN}_{7} \mathrm{O}_{4} \mathrm{~S} \\
3=\left[\mathrm{Ag}(\text { phen })\left(\mathrm{PETSC}_{4}\right)\left(\mathrm{NO}_{3}\right) \cdot \mathrm{HCl} \cdot 2 \mathrm{H}_{2} \mathrm{O}\right. \\
\mathrm{C}_{21} \mathrm{H}_{25} \mathrm{AgClN} \mathrm{O}_{7} \mathrm{O}
\end{gathered}
$$

$1=2.4 \pm 0.4(4.0 \pm 0.6)$

$2=2.3 \pm 0.3(3.8 \pm 0.5)$

$3=2.6 \pm 0.2(4.0 \pm 0.4)$
Copper(I) and silver(I) complexes of anthraldehyde thiosemicarbazone: Synthesis, structure elucidation, in vitro anti-tuberculosis/cytotoxicity activity and interactions with DNA/HSA, Khan et al. $(2020)^{25}$ 9-anthraldehyde thiosemicarbazone and phosphine
$\left.4=\mathrm{Ag}_{2}\left(\mu_{2}-\mathrm{Cl}\right)_{2}\left(\eta^{1}-\mathrm{S}-9-\text { Hanttsc }\right)_{2}\left(\mathrm{Ph}_{3} \mathrm{P}\right)_{2}\right]$

$\mathrm{C}_{68} \mathrm{H}_{56} \mathrm{Cl}_{2} \mathrm{Ag}_{2} \mathrm{~N}_{6} \mathrm{P}_{2} \mathrm{~S}_{2}$

$5=\left[\mathrm{Ag}_{2}\left(\mu_{2}-\mathrm{Br}\right)_{2}\left(\eta^{1}-\mathrm{S}-9-\text { Hanttsc }\right)_{2}\left(\mathrm{Ph}_{3} \mathrm{P}\right)_{2}\right]$ $\mathrm{C}_{68} \mathrm{H}_{56} \mathrm{Br}_{2} \mathrm{Ag}_{2} \mathrm{~N}_{6} \mathrm{P}_{2} \mathrm{~S}_{2}$

$9=\left[\mathrm{Ag}_{2} \mathrm{Cl}_{2}\left(\mu_{2}-\mathrm{S}-9-\text { Hanttsc-N }-\mathrm{Me}\right)_{2}\left(\mathrm{Ph}_{3} \mathrm{P}\right)_{2}\right]$ $\mathrm{C}_{70} \mathrm{H}_{60} \mathrm{Cl}_{2} \mathrm{Ag}_{2} \mathrm{~N}_{6} \mathrm{P}_{2} \mathrm{~S}_{2}$

$\left.10=\mathrm{Ag}_{2} \mathrm{Br}_{2}\left(\mu_{2}-\mathrm{S}-9-\text { Hanttsc-N }-\mathrm{Me}\right)_{2}\left(\mathrm{Ph}_{3} \mathrm{P}\right)_{2}\right]$ $\mathrm{C}_{70} \mathrm{H}_{60} \mathrm{Br}_{2} \mathrm{Ag}_{2} \mathrm{~N}_{6} \mathrm{P}_{2} \mathrm{~S}_{2}$

$2=\left[\mathrm{N}_{3} \mathrm{P}_{3}(\mathrm{NHCy})_{6}\left\{\mathrm{AgPPh}_{3}\right\}_{2}\right](\mathrm{TfO})_{2}$

$3=\left[\mathrm{N}_{3} \mathrm{P}_{3}(\mathrm{NHCy})_{6}\{\mathrm{AgPPh} 3\}_{3}\right](\mathrm{TfO})_{3}$

$4=\left[\mathrm{N}_{3} \mathrm{P}_{3}(\mathrm{NHCy})_{6}\{\mathrm{AgPPh} 2 \mathrm{Me}\}_{2}\right](\mathrm{TfO})_{2}$

$5=\left[\mathrm{N}_{3} \mathrm{P}_{3}(\mathrm{NHCy})_{6}\{\mathrm{AgPPh} 2 \mathrm{Me}\}_{3}\right](\mathrm{TfO})_{3}$ $6=\left[\mathrm{N}_{3} \mathrm{P}_{3}(\mathrm{NHCy})_{6}\{\mathrm{AgTPA}\}_{3}\right](\mathrm{TfO})_{3}$ $9=\left[\mathrm{N}_{3} \mathrm{P}_{3}(\mathrm{NHCy})_{3}\left(\mathrm{NMe}_{2}\right)_{3}\left\{\mathrm{AgPPh}_{2} \mathrm{Me}\right\}_{2}\right](\mathrm{TfO})_{2}$

$10=\left[\mathrm{N}_{3} \mathrm{P}_{3}(\mathrm{NHCy})_{3}\left(\mathrm{NMe}_{2}\right)_{3}\left\{\mathrm{AgPPh}_{2} \mathrm{Me}\right\}_{3}\right]$ $(\mathrm{TfO})_{3}$

Antibacterial activities and antiproliferative assays over a tumor cells panel of a silver complex with 4-aminobenzoic acid: Studies in vitro of sustained release using bacterial cellulose membranes as support, Aquaroni et al. $(2020)^{27}$

Synthesis, characterization, DFT modeling and in vitro antimycobacterial activity assays of a silver(I)-isoniazid complex, Paris Junior et al. $(2021)^{28}$

Chemical, spectroscopic characterization, molecular modeling and antibacterial activity assays of a silver (I) complex with succinic acid, Paris Junior et al. $(2021)^{29}$

Promising Ag(I) complexes with $N$-acylhydrazones from aromatic aldehydes and isoniazid against multidrug resistance in tuberculosis, Santos et al. $(2021)^{30}$ (amino)cyclophosphazenes and phosphine

\begin{tabular}{l}
$4=1.6(\mathrm{n} / \mathrm{s})^{\dagger \dagger}$ \\
$5=1.6(\mathrm{n} / \mathrm{s})$ \\
$9=12.5(\mathrm{n} / \mathrm{s})$ \\
$10=12.5(\mathrm{n} / \mathrm{s})$ \\
\hline $2=\mathrm{n} / \mathrm{s}(3.9)^{\dagger}$ \\
$3=\mathrm{n} / \mathrm{s}(0.97)$ \\
$4=\mathrm{n} / \mathrm{s}(3.9)$ \\
$5=\mathrm{n} / \mathrm{s}(3.9)$ \\
$6=\mathrm{n} / \mathrm{s}(3.9)$ \\
$9=\mathrm{n} / \mathrm{s}(3.9)$ \\
$10=\mathrm{n} / \mathrm{s}(3.9)$
\end{tabular}

$10=\mathrm{n} / \mathrm{s}(3.9)$
$12.7(28.5 \pm 6.6)$
4-aminobenzoic acid, $\mathrm{C}_{7} \mathrm{H}_{7} \mathrm{NO}_{2}$
$\left[\mathrm{Ag}\left(\mathrm{C}_{7} \mathrm{H}_{7} \mathrm{NO}_{2}\right)_{2}\left(\mathrm{NO}_{3}\right)\right]$

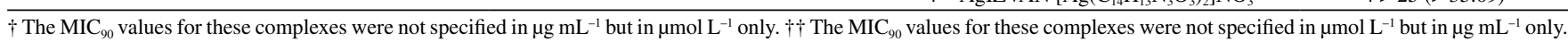
$\mathrm{n} / \mathrm{s}=$ not shown. ${ }^{*}$ IZSAL = isoniazid with salicylaldehyde; IZoVA = isoniazid with o-vanillin; IZmVA = isoniazid with $\mathrm{m}$-vanillin; IZVAN = isoniazid with vanillin. Antitubercular assays were performed against M. tuberculosis H37Rv strain.

O-atoms ${ }^{12,18-20,23,24,29}$ of the ligands, and in a number of complexes to S-13,15,17,21,24,25 and P-atoms ${ }^{15,21,25,26}$ in the case of mixed ligands. In some cases, $\mathrm{Ag}(\mathrm{I})$ is coordinated to the halides $\mathrm{Cl}^{-}$and $\mathrm{Br}^{-21,25}$ or $\mathrm{NO}_{3}{ }^{-} \cdot{ }^{14,22,27}$ This means that $\mathrm{Ag}(\mathrm{I})$ is an intermediate type of acid according to the Pearson acid-base concepts.
It is important to register that the majority of the substances used as ligands without activity against $M$. tuberculosis became active when complexed to $\operatorname{Ag}(\mathrm{I}) .{ }^{14,16-18,20-27,29}$ Four works show higher $\mathrm{MIC}_{90}$ values for the $\mathrm{Ag}$-complexes than their respective ligands. This is the case for 6 -mercaptopurine, ${ }^{13}$ and the already 
commercial drugs against TB: isoniazid ${ }^{28}$ (and $\mathrm{N}$-acylhydrazones + isoniazid) ${ }^{30}$ and the second-line drug cycloserine. ${ }^{19}$ Two works did not give the $\mathrm{MIC}_{90}$ values for the free ligands acesulfame ${ }^{12}$ and tautomeric thiazolidine-2-thione. ${ }^{15}$

The first-line drug isoniazid appears as a ligand in one work but the $\mathrm{MIC}_{90}$ value of the complex is higher than the free drug. ${ }^{28}$ In another work, isoniazid appears associated to phenanthroline forming a ligand that is a Schiff base. ${ }^{12}$ A work published in 2021 presents four complexes containing isoniazid associated to $N$-acylhydrazones derivatives. ${ }^{30}$

Mechanisms of action of $\mathrm{Ag}(\mathrm{I})$ complexes related to M. tuberculosis are not well described in the literature but there are some evidences of interaction of some $\operatorname{Ag}(\mathrm{I})$ complexes with DNA. There are four possible mechanisms proposed for inhibition of bacteria growth by aqueous $\mathrm{Ag}(\mathrm{I})$ ions: (i) interference with the electron transport system, (ii) DNA-binding, (iii) interaction with the cell membrane, ${ }^{12,13}$ and (iv) interaction with the thiol group in the vital enzymes to inactivate them. ${ }^{15}$

Considering the $\mathrm{MIC}_{90}$, it can be concluded that a number of complexes have values that recommended them as antimicrobial agents in the future, because these $\mathrm{Ag}$-complexes present lower $\mathrm{MIC}_{90}$ values than the second-line drugs such as ofloxacin, levofloxacin, and moxifloxacin. ${ }^{30-32}$

Table 4 highlights the $\mathrm{MIC}_{90}$ against $M$. tuberculosis of the drugs most commonly used in the fight against TB.

Table 4. Selected $\mathrm{MIC}_{90}$ of first- and second-line drugs against $M$. tuberculosis H37Rv strain

\begin{tabular}{lcc}
\hline Substance & $\begin{array}{c}\text { Average } \mathrm{MIC}_{90} \text { and } \\
\text { standard deviation } \\
\left(\mu \mathrm{gL}^{-1}\right)\end{array}$ & $\left.\mathrm{MIC}_{90}(\mu \mathrm{mol} \mathrm{L})^{-1}\right)$ \\
\hline First line & $<0.098$ & 0.715 \\
$\quad$ Isoniazid & $<0.098$ & 0.119 \\
Rifampicin & $1-2$ & $\mathrm{n} / \mathrm{s}$ \\
Ethambutol & $6-50$ & $\mathrm{n} / \mathrm{s}$ \\
$\quad$ Pyrazinamide & & \\
\hline Second line & $0.276 \pm 0.091$ & 0.475 \\
Streptomycin & $0.382 \pm 0.169$ & 1.057 \\
Ofloxacin & $0.561 \pm 0.165$ & 0.958 \\
Amikacin & $0.625 \pm 0.024$ & 1.557 \\
Moxifloxacin & $16-32$ & $\mathrm{n} / \mathrm{s}$ \\
Amoxicilin &
\end{tabular}

$\mathrm{MIC}_{90}=$ Minimum concentration that inhibits $90 \%$ of bacterial growth $\mathrm{n} / \mathrm{s}=$ not shown. Adapted from Santos et al. $(2021$, p. 4$),{ }^{30}$ Horita et al. (2014, p. 7011$)^{31}$ and Pyrazinamide (2008, p. 141-142). ${ }^{32}$

It is possible to list as the most effective $\operatorname{Ag}(\mathrm{I})$ complexes against $M$. tuberculosis the ones with two ligands, one of them being phosphine $\left(\mathrm{PPh}_{3}\right)$, in three works: Khan et al. $(2018)^{21}$ (5 complexes with $\mathrm{MIC}_{90}$ value $\left.1.6 \mu \mathrm{g} \mathrm{mL} \mathrm{m}^{-1}\right)$, Khan et al. ${ }^{25}$ (2 complexes with $1.6 \mu \mathrm{g} \mathrm{mL}^{-1}$ ), and Gascón et al. ${ }^{26}$ (1 complex with $2.2 \mu \mathrm{g} \mathrm{mL} \mathrm{m}^{-1}$, $\left.0.97 \mu \mathrm{mol} \mathrm{L}{ }^{-1}\right)$. The complexes described by Silva et al.,${ }^{24}$ on the other hand, were synthesized with phenantroline and thiosemicarbazones (0.97-3.9 $\left.\mu \mathrm{mol} \mathrm{L}{ }^{-1}\right)$. Exception to the complex by Massabni et al. ${ }^{28}$ $\left(0.8 \mu \mathrm{g} \mathrm{mL} \mathrm{m}^{-1}, 2.6 \mu \mathrm{mol} \mathrm{L} \mathrm{L}^{-1}\right)$ that used isoniazid as ligand but shows less effective results than the free ligand.

This review did not present articles that show research on silver nanoparticles against $M$. tuberculosis although the bactericidal effect of $\mathrm{Ag}$ nanoparticles has been well known.

\section{CONCLUSIONS}

Tuberculosis has been one of the deadliest diseases worldwide for ages. Anti-TB treatments have been efficient, but new drugs and alternative treatments are needed to reduce the number of people infected by this disease mainly because of the occurrence of multidrug resistant TB strains bacteria. The standard treatments basically use four first-line drugs: isoniazid, rifampicin, pyrazinamide and ethambutol. Despite the efficiency of these drugs, used since 1960, or their combination with other drugs, the cases of TB deaths continue to grow. Second-line drugs have also been used for the same reasons. This line includes drugs of the fluoroquinolones and aminoglycosides classes.

Metal complexes are presented as alternatives/options for antiTB treatments. There are no cases of TB treatments using metal complexes but research in this area shows that they could be important and used in the future as adjuvants in eradicating the bacteria that contaminate environments and many surgical materials and objects. Silver is not considered an essential metal for living organisms. On the contrary, it is considered toxic, but many new complexes have been prepared and tested for use against diseases such as tuberculosis, cancer, leishmaniasis and others, and also to prevent contamination by bacteria in cases of severe burns.

In the present work, 19 articles were selected and $55 \mathrm{Ag}$ complexes that have proven to be efficient against the TB bacillus were analyzed. Many of them described synthesis and structural features involving Ag-complexes with various types of ligands. In general, the complexes showed greater efficiency than the organic molecules used as ligands.

There are suggestions for $\mathrm{Ag}(\mathrm{I})$ complex action by comparison with other metal ions, including $\mathrm{Pt}$ (II). The mechanisms of action are not well elucidated, but there are good prospects for further biological studies of the action of the complexes. So they can be used as drugs in the future for the treatment of TB, cancer and other diseases.

\section{ACKNOWLEDGEMENTS}

This article is dedicated to Dr. Eduardo Henrique Bonini, in memoriam, for his commitment to the treatment of tuberculosis.

\section{REFERENCES}

1. Word Health Organization (WHO); Global Tuberculosis Report 2018, WHO: Geneva, 2018. available at https://apps.who.int/iris/bitstream/han dle/10665/274453/9789241565646-eng.pdf?sequence=1\&isAllowed=y, accessed in August 2021.

2. Zumla, A.; Chakaya, J; Centis, R.; D'Ambrosio, L.; Mwaba, P.; Bates, M.; Kapata, N.; Nyirenda, T.; Chanda, D.; Mfinanga, S.; Hoelscher, M.; Maeurer, M.; Migliori, G. B.; Lancet Respir. Med. 2015, 3, 220.

3. Pai, M.; Behr, M. A.; Dowdy, D.; Dheda, K.; Divangahi, M.; Boehme, C. C.; Ginsberg, A.; Swaminathan, S.; Spigelman, M.; Getahun, H.; Menzies, D.; Raviglione, M.; Nat. Rev. Dis. Primers 2016, 2, 16076.

4. Rabahi, M. F.; Silva Júnior, J. L. R.; Ferreira, A. C. G.; Tannus-Silva, D. G. S.; Conde, M. B.; J. Bras. Pneumol. 2017, 43, 472.

5. Word Health Organization (WHO); WHO treatment guidelines for drug-resistant tuberculosis, 2016 update. October 2016 revision, WHO: Geneva, 2016. Available at http://apps.who.int/iris/bitstream/hand le/10665/250125/9789241549639-eng.pdf;jsessionid=FC8D03A3974 3D8BA63EFDAF2A9EFE213? sequence=1, accessed in August 2021.

6. Ventola, C. L.; P and T 2015, 40, 277.

7. Santos, D. P.; Souza, D. V.; Rodrigues, S.; Avelar, K. E. S.; Revista de Saúde 2012, 3, 13.

8. Brazil; Ministério da Saúde; Secretaria de Vigilância em Saúde; Departamento de Vigilância das Doenças Transmissíveis; Manual de 
Recomendações para o Controle da tuberculose no Brasil, Ministério da Saúde: Brasília, 2018.

9. Medici, S.; Peana, M.; Nurchi, V. M.; Zoroddu, M. A.; J. Med. Chem. 2019, 62, 5923.

10. Whittemore, R.; Knafl, K.; J. Adv. Nurs. 2005, 52, 546

11. Stovold, E.; Beecher, D.; Foxlee, R.; Noel-Storr, A.; Syst. Rev. 2014, 3, 54.

12. Cavicchioli, M.; Massabni, A. C.; Heinrich, T. A.; Costa-Neto, C. M.; Abrão, E. P.; Fonseca, B. A. L.; Castellano, E. E.; Corbi, P. P.; Lustri, W. R.; Leite, C. Q. F.; J. Inorg. Biochem. 2010, 104, 533.

13. Cuin, A.; Massabni, A. C.; Pereira, G. A.; Leite, C. Q. F.; Pavan, F. R.; Sesti-Costa, R.; Heinrich, T. A.; Costa-Neto, C. M.; Biomed. Pharmacother. 2011, 5, 334.

14. Pereira, G. A.; Massabni, A. C.; Castellano, E. E.; Costa, L. A. S.; Leite, C. Q. F.; Pavan, F. R.; Cuin, A.; Polyhedron 2012, 38, 291.

15. Altaf, M.; Stoeckli-Evans, H.; Cuin, A.; Sato, D. N.; Pavan, F. R.; Leite, C. Q. F.; Ahmad, S.; Bouakka, M.; Mimouni, M.; Khardli, F. Z.; Hadda, T. B.; Polyhedron 2013, 62, 138.

16. Paiva, I. L.; Carvalho, G. S. G.; Silva, A. D.; Corbi, P. P.; Bergamini, F. R. G.; Formiga, A. L. B.; Diniz, R.; Carmo, W. R.; Leite, C. Q. F.; Pavan, F. R.; Cuin, A.; Polyhedron 2013, 62, 104.

17. Segura, D. F.; Netto, A. V. G.; Frem, R. C. G.; Mauro, A. E.; Silva, P. B.; Fernandes, J. A.; Paz, F. A. A.; Dias, A. L. T.; Silva, N. C.; Almeida, E. T.; Marques, M. J.; Almeida, L.; Alves, K. F.; Pavan, F. R.; Souza, P. C.; Barros, H. B.; Leite, C. Q. F.; Polyhedron 2014, 79, 197.

18. Silva, S. A.; Leite, C. Q. F.; Pavan, F. R.; Masciocchi, N.; Cuin, A.; Polyhedron 2014, 79, 170.

19. Ciol, M. R.; Manzano, C. M.; Cuin, A.; Pavan, F. R.; Ribeiro, C. M.; Ruiz, A. L. T. G.; Oliveira, E. C. S.; Lustri, W. R.; Fregonezi, N. F.; Nogueira, F. A. R.; Corbi, P. P.; ChemistrySelect 2018, 3, 1719.

20. Amaral, T. C.; Miguel, F. B.; Couri, M. R. C.; Corbi, P. P.; Carvalho, M. A.; Campos, D. L.; Pavan, F. R.; Cuin, A.; Polyhedron 2018, 146, 166.

21. Khan, A.; Jasinski, J. P.; Smolenski, V. A.; Hotchkiss, E. P.; Kelley, P. T.; Shalit, Z. A.; Kaur, M.; Paul, K.; Sharma, R., Bioorg. Chem. 2018, 80,303 .
22. Ahmed, M.; Rooney, D.; McCann, M.; Devereux, M.; Twamley, B.; Galdino, A. C. M.; Sangenito, L. S.; Souza, L. O. P.; Lourenço, M. C.; Gomes, K.; Santos, A. L. S.; BioMetals 2019, 32, 671.

23. Manzano, C. M.; Nakahata, D. H.; Tenorio, J. C.; Lustri, W. R.; Nogueira, F. A. R.; Aleixo, N. A.; Gomes, P. S. S.; Pavan, F. R.; Grecco, J. A.; Ribeiro, C. M.; Corbi, P. P.; Inorg. Chim. Acta 2020, 502, 119293.

24. Silva, D. E. S.; Becceneri, A. B.; Solcia, M. C.; Santiago, J. V. B.; Moreira, M. B.; Gomes Neto, J. A.; Pavan, F. R.; Cominetti, M. R.; Pereira, J. C. M.; Netto, A. V. G.; Dalton Trans. 2020, 49, 5264.

25. Khan, A.; Paul, K.; Singh, I.; Jasinski, J. P.; Smolenski, V. A.; Hotchkiss, E. P.; Kelley, P. T.; Shalit, Z. A.; Kaur, M.; Banerjee, S.; Roy, P.; Sharma, R.; Dalton Trans. 2020, 49, 17350.

26. Gascón, E.; Maisanaba, S.; Otal, I.; Valero, E.; Repetto, G.; Jones, P. G.; Jiménez, J.; Inorg. Chem. 2020, 59, 2464.

27. Aquaroni, N. A. S.; Nakahata, D. H.; Lazarini, S. C.; Resende, F. A.; Cândido, A. L. P.; Barud, H. S.; Claro, A. M.; Carvalho, J. E.; Ribeiro, C. M.; Pavan, F. R.; Lustri, B. C.; Ribeiro, T. R. M.; Moreira, C. G.; Cândido, T. Z.; Lima, C. S. P.; Ruiz, A. L. T. G.; Corbi, P. P.; Lustri, W. R.; J. Inorg. Biochem. 2020, 212, 111247.

28. Paris Junior, J. A.; Cavicchioli, M.; Machado, R. T. A.; Pavan, F. R.; Nakahata, D. H.; Corbi, P. P.; Costa, A. M. F.; Pereira, D. H.; Massabni, A. C.; Quim. Nova 2021, 44, 278.

29. Paris Junior, J. A.; Rocchi, A. J. S.; Biagioni, B. T.; Cavicchioli, M.; Machado, R. T. A.; Pavan, F. R.; Corbi, P. P.; Lustri, W. R.; Pereira, D. H.; Massabni, A. C.; Eclet. Quim. J. 2021, 46, 26.

30. Santos, P. V. P.; Ribeiro, C. M.; Pavan, F. R.; Corbi, P. P.; Bergamini, F. R. G.; Carvalho, M. A.; D’Oliveria, K. A.; Cuin, A. J.; Mol. Struct. 2021, 1234, 130193.

31. Horita, Y.; Maeda, S.; Kazumi, Y.; Doi, N.; Antimicrob. Agents Chemother. 2014, 58, 7010.

32. Pyrazinamide, Tuberculosis 2008, 88,141 . 